\title{
The Exact Velocity in Low-redshift Stationary Radio Sources from its Apparent Longitudinal and Transverse Velocity
}

\author{
Wing Tang
}

\section{Abstract}

While the term Apparent Transverse Velocity (ATV) is popularly used to explain some super-luminal (faster-than-light) apparent motions in distant astronomical objects, we ask whether the Apparent Longitudinal (radial) Velocity (ALV), which has never been mentioned in astronomical literature hitherto, can be embedded and meaningfully considered. In this paper we promote the idea of ALV; combining ALV and ATV theoretically allows us to determine precisely the exact velocity of the observable jets from the very low-redshift sources which are stationary relative to the Earth's observer. The measurement and calculation of ALV are also proposed and discussed.

\section{Introduction}

Many active galactic nuclei (AGN), and even microquasars nowadays, contain the compact sources of radio emission with components that appear to move apart in successive very long baseline interferometry (VLBI) images. The ATV of the separation of some components exceeds the speed of light (c); such motion and sources are called superluminal. This phenomenon has become common ever since the VBLI technique was invented. It is interesting to note that the radiation from these sources is widely believed to be a result of the accretion of mass into the supermassive black hole located at the centre of them. Figure 1 shows how the radio-loud AGN looks like, and Figure 2 compares the quasars and microquasars.

However the existence of black holes inside the AGN remains yet to be confirmed, the observed superluminal motion might appear to contradict 
special relativity because it violates causality. They have no such contradiction, yet detecting such motion even supportsthe theory that quasars, BL Lac objects and radio galaxies may just be the same, known as active galaxies viewed from different perspectives (Biretta, Sparks, \& Macchetto 1999). In 1966, Martin Rees predicted such motion before the observation was made. Nowadays, the popular explanation for superluminal motion is the visual result of light travel-time effects resulting from the finiteness of $c$ (Shu 1982). In details, the AGN contain a nearly straight, expanding jet of plasma moving relativistically (still below the speed of light $(c)$ ). The superluminal radio sources are modelled by the radiating blobs that move at the relativistic speed $v$ away from the AGN core. If the jet points close to the line of sight, contraction of the apparent timescale between the blobs at different times can give rise to superluminal motion - the apparent transverse velocity that is greater than $c$. Such apparent timescale contraction can be visualized in Figure 3 and be later explained.

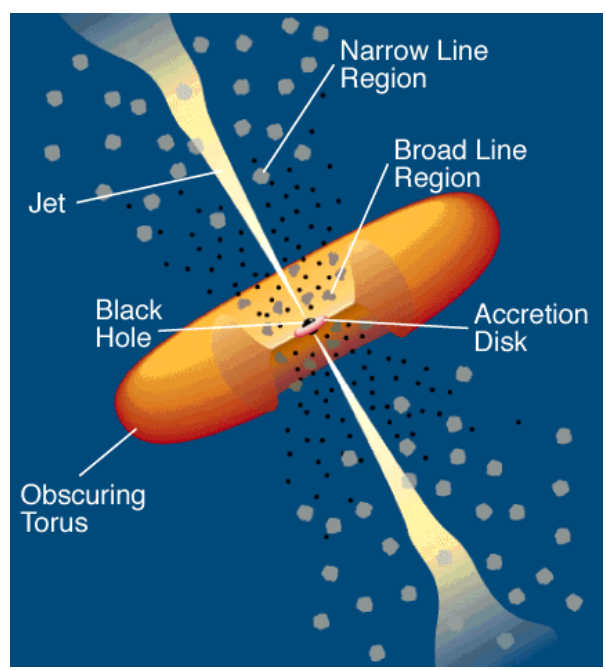

Figure 1. Radio-Loud AGN Model

From: Urry, C.M., \& Padovani, P. (2003). Radio-Loud AGN Model. Retrieved 1st November, 2011, from NASA's HEASARC: Education \& Public Information http://heasarc.nasa.gov/docs/objec ts/agn/agn model.html

But knowing the ATV is not precise enough. Since the exact velocity of astronomical objects (relative to the source) is at least of theoretical importance, and might help us understand physics of the accretion-powered jets and the black hole itself, we desire a way of accurately calibrating them; but a quick look at the astronomical literature tells us that the exact speed and the angle to the line of sight are not so accurately determined (Shu 1982). Therefore, we aim to provide another (maybe better) way to determine the exact speed $v$.

In our paper, we study the low-redshift radio sources whose AGN core is almost stationary relative to us. The reason for this is its simplicity, and studying the nearby microquasars is important for the study of relativistic jets. We know that 
the jets are formed near the compact region, and the timescales near the compact region increase with the mass of the compact region. Hence microquasars might take only one day to go through all the variations in the quasars that take centuries, which means that we can see a more complete evolution of the relativistic jet system.

We first introduce and derive the ATV $v_{a p p, t}$, and give the standard determination of $\mathrm{v}$. Then we introduce the ALV $v_{a p p, l}$ from the head-on movement into the non-head-on movement. Later we talk about how to make use of our theory with current observational methods to obtain $v$. The condition "low-redshift stationary radio sources" may not be physical and general, since most superluminal motions are observed in highredshift radio sources that the AGN core probably moves at nearly the speed of light (Chodorowski 2005). But one such simplified case can be a good starting point to consider how $v_{a p p, l}$ complements to determine $\mathrm{v}$, and can be embedded in our literature.

In future, we would follow Michal J. Chodorowski's approach to generalize all such considerations for all arbitrary $z$ and $v \in[0, c]$ so that the theory can be entirely physical and generalized to various astronomical radio sources that move relativistically relative to us (Chodorowski 2005).
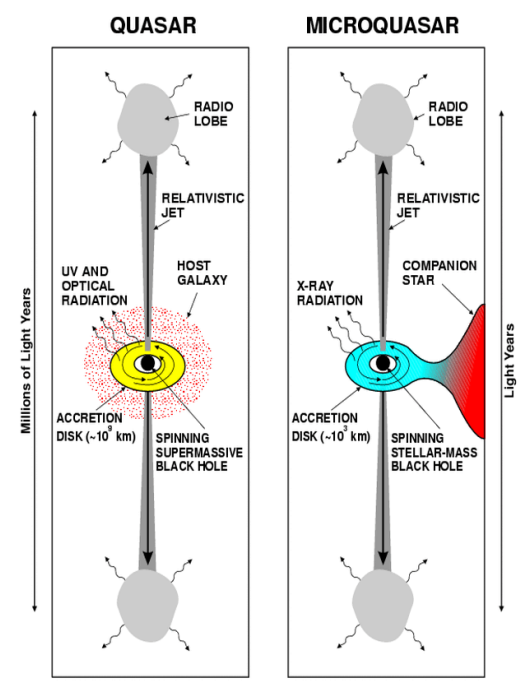

Figure 2. A diagram illustrating current ideas about quasars and microquasars

From: Mirabel, I.F., \& Rodriguez, L.F. (1998), Nature, 392, 673. Retrieved 1st November, 2011, from http:// www.nature.com/nature/journal/v392/n6677/fig tab/392673a0 Fl.html 


\section{ATV}

In astronomical literature, the superluminal motions are widely explained using the term ATV. The derivation can leave us a hint of how to define and derive the ALV $v_{a p p, l}$, later. In this section, we can also see the deficiency of the current standard method of determining $v$.

\section{The Derivation of ATV}

Figure 3 shows the schematic diagram of how we visually perceive the movement of an object. We consider the object (imagined as the radio source) moving with a velocity $v$ from $P_{1}$ to $P_{2}$ in a time $\Delta t$ in the observer's frame; so, the length $l=v \Delta t$.

Suppose the object is continuously emitting/reflecting light so that their entire body is visible. Let photons be emitted from $P_{1}$ and $P_{2}$ at times $t_{1}$ and $t_{2}$, respectively, with the time difference

$\Delta t=t_{2}-t_{1}$

Although the AGN core is at redshift $z(1$, we assume $D) l$ for the entire observing time duration T. So the lines of sight, $L_{1}$ and $L_{2}$, from $P_{1}$ and $P_{2}$ respectively, can be approximately parallel. The times at which these photons are received by the observer are given by:

$$
\begin{gathered}
t_{1}^{r e c}=t_{1}+\frac{D+v \Delta t \cos \theta}{c} \\
t_{2}^{r e c}=t_{2}+\frac{D}{c}=t_{1}+\Delta t+\frac{D}{c}
\end{gathered}
$$

The apparent time interval is

$$
\Delta t^{r e c}=t_{2}^{r e c}-t_{1}^{r e c}=\Delta t\left(1-\mid \frac{v}{c} \cos \theta\right)
$$

The actual transverse distance moved by the object is

$$
l_{\perp}=v \Delta t \sin \theta
$$

Hence the ATV $V_{a p p, t}$ of the object is given by

$$
v_{a p p, t}=\frac{l_{\perp}}{\Delta t^{r e c}}=\frac{v \sin \theta}{1-\frac{v}{c} \cos \theta}
$$




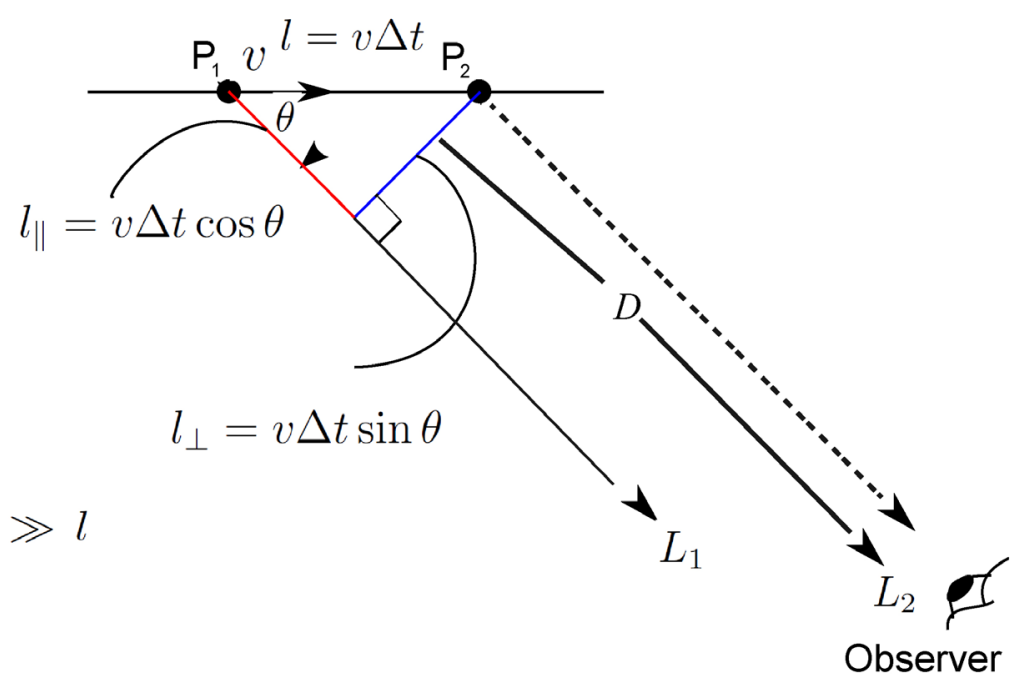

Figure 3. The apparent transverse movement seen by the ground observer

With $\beta=v / c$ and $\beta_{a p p, t}=v_{a p p, t} / c$, the ATV can be equivalently written as $\beta_{\text {app }, \text { : }}$

$$
\beta_{a p p, t}=\frac{\beta \sin \theta}{1-\beta \cos \theta}
$$

\section{Superluminal Motions and Determination of $\beta$ and $\theta$}

The value of $\beta_{a p p, t}$ can be obtained in observation as shown in Figure 4 . In reality, the measurement of angle $\theta$ is never simple; therefore, $\beta$ cannot be simply obtained by only Eq. (7). Our current idea of finding $\theta$ and $\beta$ is first determine the angle for which the apparent velocity is a maximum:

$$
\frac{d \beta_{a p p, t}}{d \theta}=\frac{\beta(\cos \theta-\beta)}{(1-\beta \cos \theta)^{2}}
$$

It is assumed that $\beta<1$. For the approaching source, the angle $\theta$ satisfies $0 \leq \theta$ $\leq \pi / 2$. Hence. 
1. $\beta_{\text {app } t} / d \theta>0$ happens if $\theta<$ $\cos ^{-1} \beta$;

2. $\beta_{\text {app } t} / d \theta<0$ happens if $\theta>$ $\cos ^{-1} \beta$.

If the object is in uniform motion from the angle $\theta=0$ to $\pi / 2$, then the object is first seen to be accelerating towards us until $\cos \theta=\beta$, but decelerating afterwards. Hence $\beta$ app, $t$ reaches a maximum when $\cos \theta=\beta$ and so we have

$$
\beta_{a p p, t ; \max }=\frac{\beta \sin \theta}{1-\beta \cos \theta}=\frac{\beta \sqrt{1-\beta^{2}}}{1-\beta^{2}}=\frac{\beta}{\sqrt{1-\beta^{2}}}=\Gamma \beta
$$

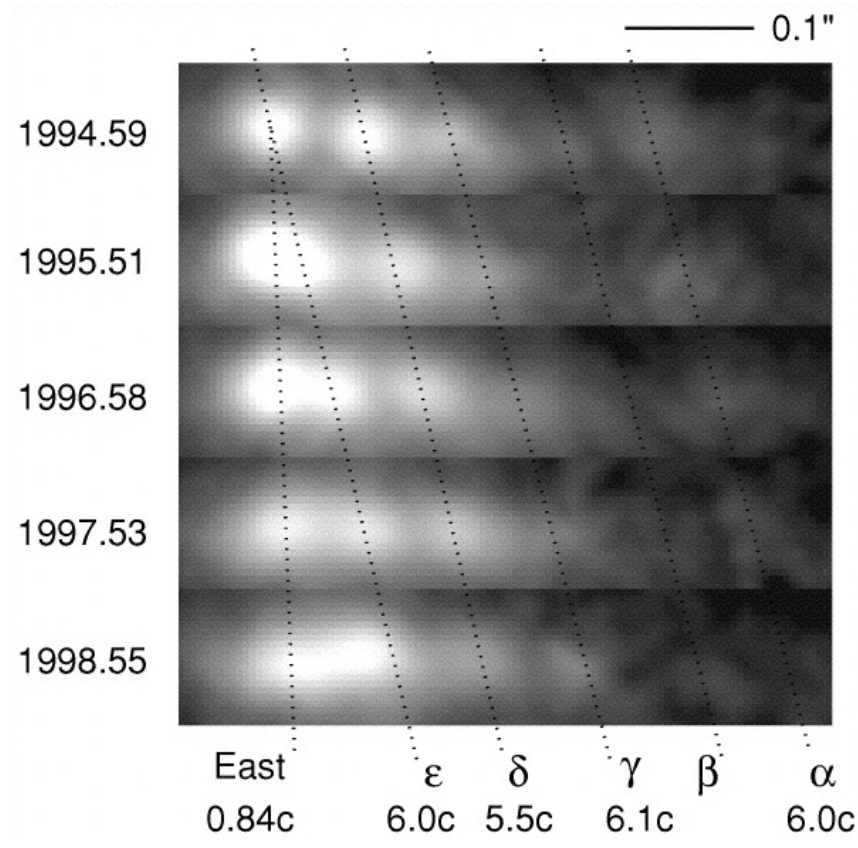

Figure 4. Sequence of $\mathbf{F} 342 \mathrm{~W}(\gamma=\mathbf{3 4 2} \mathrm{nm})$ images showing evolution of some components in one region of the M87 jet between 1994 and 1998. The dotted lines are to identify and track features between epochs and are labeled with component names and speeds derived

From: Biretta, Sparks, \& Macchetto (1999). ApJ, 520, 621. Retrieved 1st November 2011, from IOP Science, http://iopscience.iop.org/0004-637X/520/2/621/39787.fg2.html 
If $\Gamma \gg 1$, then $\beta \approx 1$ and so $\beta_{\text {app, } t \text {; } \max } \gg 1$, i.e. the maximum ATV can be much greater than the speed of light. This explains the possibility of superluminal motions. For example, when $\theta=\pi / 4$ and $\beta=0: 9$ and $\cos \pi / 4=1 / \sqrt{ } 2 \neq 0.9$, we have $\beta_{\text {app }, t}=1.75>1$.

Now using Eq. (9), we can obtain the lower bound of $\beta$ without the knowledge of $\theta$ :

$$
\begin{aligned}
\frac{\beta}{\sqrt{1-\beta^{2}}}=\beta_{a p p, t ; \max } & \geq \beta_{a p p, t} \\
\Rightarrow \beta & \geq \frac{\beta_{a p p, t}}{\sqrt{1+\beta_{a p p t}^{2}}}
\end{aligned}
$$

The equality holds if and only if $\beta_{a p p, t}$ reaches the maximum over [0, Л/2]. Вy plotting the variation of $\beta_{a p p, t}$, we can spot the maximum $\beta_{a p p, t ; \max }$ and then obtain the value $\beta$ by Eq. (10). Using $\cos \theta=\beta$ with the determined $\beta$, we can obtain the actual angle $\theta$ when the maximum occurs.

\section{Introduction to $\mathrm{ALV}$}

Although seeking the maximum $\beta_{a p p, t ; \max }$ it can work well to obtain the values of $\theta$ and $\beta$, the maximum $\beta_{a p p, t \text {; } m a x}$ (for some distant astronomical objects moving close to the line of sight) may not be reached in our human lifespan because the proper motion (the angular movement) of distant objects is so small; moreover, the actual motion is usually non-uniform; if the apparent time interval is taken to be too long, the speed we obtain is not representative at all and might make us miss out important information about the dyanmics of the jet.

To obtain the actual speed $v$ and $\beta$, we consider if it is possible to obtain the angle $\theta$ using any simple idea. A naive idea, known from secondary school trigonometry, is if we can have the knowledge of the apparent velocity $u$ that is perpendicular to the ATV, then we can determine the angle using

$$
u \tan \theta=v_{a p p, t}
$$


Since $u$ is, in turn, along our line of sight, $u$ can be defined as the "apparent longitudinal velocity (ALV)" which acts in a complementary role with ATV.In principle, it should be the actual radial distance of the object divided by the apparent time interval at both ends of which the signals are received by the observer. The existence of this term has arisen without any meaning. To be concrete, we consider the following idealized case - the head-on movement.

\section{Head-on Movement}

Suppose we have a continuously light-emitting/reflecting object which is actually a non-point source. We can determine the radial velocity of the object by observing the change in the angular size of itself or some of its components. If two identical ping-pong balls are placed at distances of 1 metre and 2 metres respectively, then the farther object subtends approximately half the angle of the nearer object. Now we see that an object heading towards us is getting bigger and bigger in angular size because it is getting nearer, while an object receding from us is getting smaller and smaller in angular size because it is farther away.

Imagine when all the component particles in the jet move with the same velocity, the component's angular size changes linearly as it moves radially at a constant rate, if the physical size of the component does not change much. This rate of relative change in the angular size is equal to the relative change in distance. Nowadays the very long baseline array (VLBA) is used to measure the distance of the radio galaxy and the jet component, hence the radial velocities can be found (Dravins at al. 1999). This method is called "changing angular extent" in space astrometry which has been applied to Hipparos data (Madsen, Lindegeren, \& Dravins 1999).

In Figure 5 the object is just 1-light-second away from the observer (us), moving with $v=\mathrm{c} / 2$ towards us at $t=0$. The object first emits/reflects the red light.

At $t=1 \mathrm{~s}$, by the definition of 1-light-second, the red light emitted at $t=0$ has reached us. We perceive the angular size of the object at $t=0$, but the source is moving mid-way at our own time.

Now we take $t=1.5 \mathrm{~s}$. We perceive the angular size of the object at $t=1 \mathrm{~s}$, since the blue light emitted exactly at 1s should travel half-of-1-light-second from the midway of the separation between the object and us at $t=0$.

As an ignorant observer, we find the ALV - the actual distance 0.5cs told by the angular-size difference divided by the apparent time interval $0.5 \mathrm{~s}$ - is indeed equal to $c$. So we expect if we choose $v>c / 2$, we will have the ALV higher than $c$. 


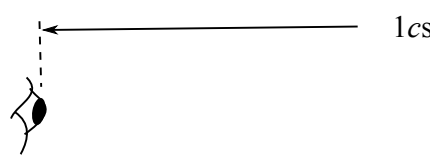

Observer

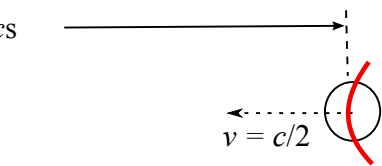

The red light emitted

(a) $t=0$

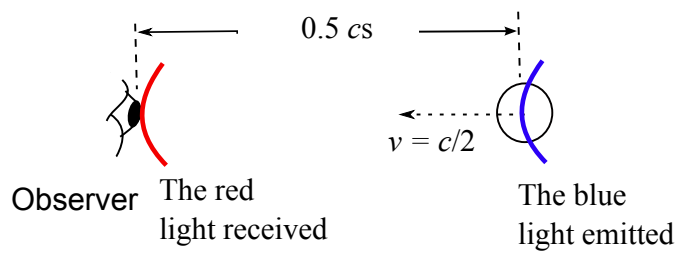

(b) $t=1 \mathrm{~s}$

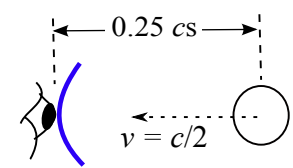

Observer The blue

light received

(c) $t=1.5 \mathrm{~s}$

Figure 5. The apparent longitudinal movement seen by the observer

\section{Non-head-on Movement}

Astronomical objects may not always go along our line of sight. They can approach, or recede from, us at the angle $\theta$ from our line of sight as shown by Figure 3. If we focus on the red line along the line of sight $L_{1}$ in Figure 3, it is the same actual distance as that in head-on movement; the apparent time interval in Eq. (4) is exactly the same as that in head-on movement. Hence the ALV $v_{a p p, l}$ is given by

$$
v_{a p p, l}=\frac{l_{\|}}{\Delta t^{r e c}}=\frac{v \cos \theta}{1-\frac{v}{c} \cos \theta}
$$

Equivalently, if $\beta_{a p p, l}:=v_{a p p, l} / c$, we can write

$$
\beta_{a p p, l}=\frac{\beta \cos \theta}{1-\beta \cos \theta}
$$


To ensure this exactly coincides with the result in head-on movement, we substitute $\theta=0$ and $\beta=1 / 2$; we see $\beta_{\text {app }, l}=1$, i.e. $v_{a p p, l}=c$. Eq. (13) also allows us to write Eq. (11) so that the angle $\theta$ can be determined. On the other hand, we observe that the actual radial velocity $v_{r}$ can be written as

$$
\beta_{r}:=\frac{v_{r}}{c}=\beta \cos \theta
$$

and be purely determined by the measured quantity $\beta_{\text {app }, l}$ :

$$
\beta_{r}=\beta \cos \theta=\frac{\beta_{\text {app }, l}}{1+\beta_{\text {app }, l}}
$$

We now square Eq. (13) and Eq. (7) individually and sum them up. We will find

$$
\beta_{\text {app }, t}^{2}+\beta_{a p p, l}^{2}=\frac{\beta^{2}}{(1-\beta \cos \theta)^{2}}
$$

Combining this with Eq. (15), we have the exact speed

$$
\beta=\frac{\sqrt{\beta_{a p p, t}^{2}+\beta_{a p p, l}^{2}}}{1+\beta_{a p p, l}}
$$

with, if $\beta \neq 0$,

$$
\theta=\arccos \left(\frac{v_{r}}{v}\right)=\arccos \left(\frac{\beta_{a p p, l}}{\sqrt{\beta_{a p p, t}^{2}+\beta_{a p p, l}^{2}}}\right)
$$

Note that in our measurement we would obtain br, and hence we can indeed find out $\beta_{a p p, l}$ via Eq. (15) and substitute this $\beta_{a p p, l}$ into Eq. (17a)-(17b).

\section{Observational Techniques Fitting our Theory}

In the previous section, a theoretical footing of how to calculate the exact velocity is obtained; however, some observational problems have to be addressed and considered. 


\section{Problems with Measurement of ALV Through its Angular Size}

We mentioned the measurement of relative change in the angular-size of the distant object can tell us the ALV. In fact, the relative change in the angular size of the distant object is microscopically small - the device nowadays cannot detect such change. So it is never feasible to obtain the knowledge of ALV through the angular size change.

For the close enough source, we might have to answer how close it has to be in order to make the analysis and measurement valid. Here we leave the quantitative answer for readers to investigate, yet we want to say that such expectation is theoretically possible. For the measurement of the radial velocities, the VLBI techniques must be used. Since the angular size is extremely small, we can use the "skinny triangle approximation" regime throughout the lifetime of the relativistic clouds.

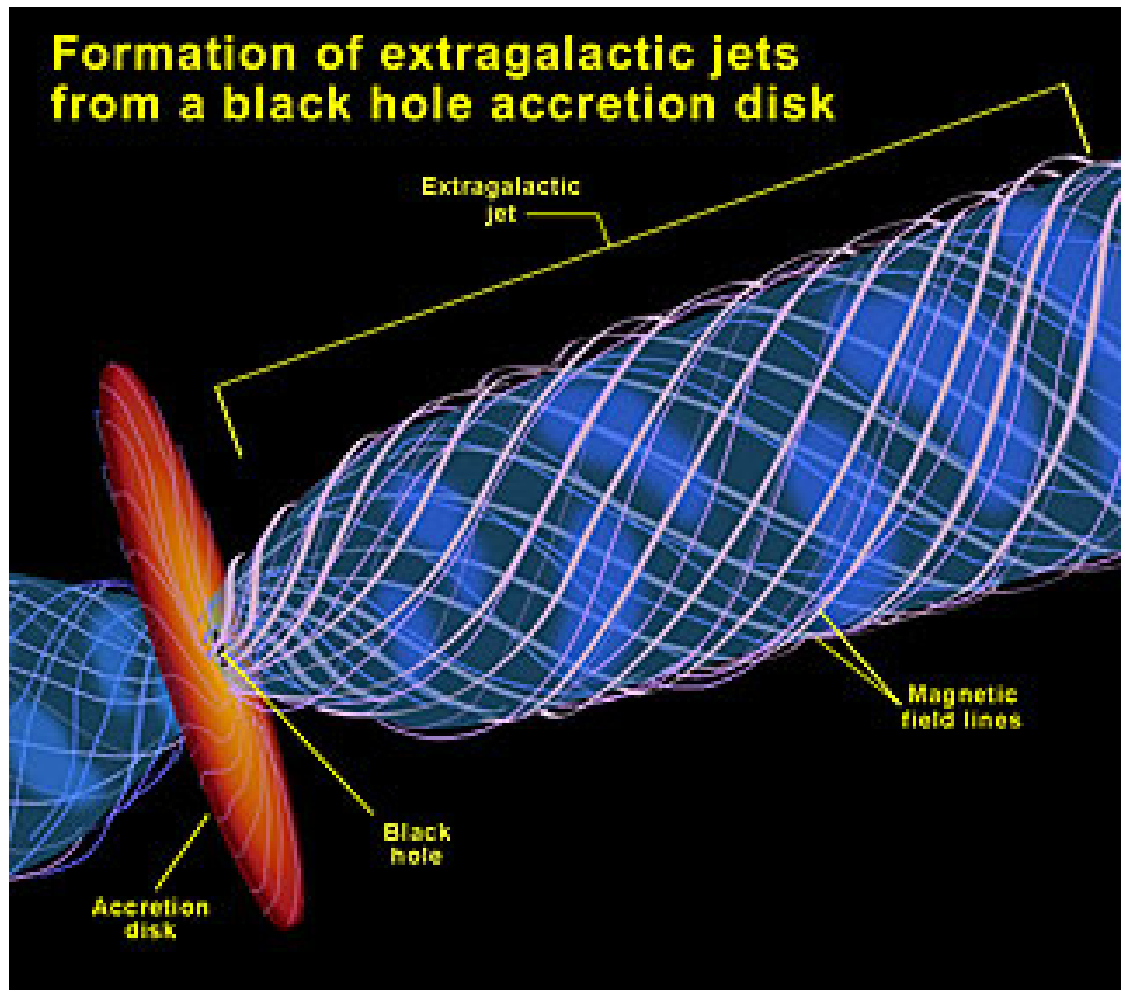

Figure 6. The imagination of a jet formation. A jet, shaped by strong, twisting field lines, blasts upwards from the accretion disk around the black hole

From: NASA/ESA and Ann Feild (1999). Formation of Extragalactic Jets from a Black Hole Accretion Disk. Retrieved 1st November 2011, from ESA/Hubble, http://www.spacetelescope.org/images/opo9943e/ 
However this measurement is possible, we have to face the following physical problem: the relativistic jets and clouds themselves actually evolve during their lifetimes as they expand and are diverted by their interactions with magnetic fields and ambient, nonrelativistic plasma in the vicinity of the AGN or microquasars as displayed by Figure 6. The evolutionary effects would be confused with the angularsize- change effects, which makes our measurement results unreliable.

\section{The Standard Method: Doppler Shift in the Spectrum of the Jets}

To detect the apparent speed of objects does not necessarily require the angularsize change. One standard way to measure the actual radial velocity $\beta_{r}$ of the objects is the Doppler shift in their spectrum. This would have to be based on the knowledge of the chemical compositions of the objects. In fact, in most of the currently known superluminal sources there are synchrotron jets from Quasars which have no visible spectral lines. But, nearby radio sources such as SS 433 sitting in our Milky Way Galaxy have shown that they have the visible atomic emission lines from the jets with the very significant Doppler shift in both optical and X-ray spectral lines. So the jet velocities can still be derived using the relativistic Doppler effect (Margon 1984).

Suppose we know the Doppler shift in their spectrum. We have had the knowledge of the source frequency $v_{S}$ and that of the observed frequency $v_{O}$ The relativistic Doppler effect tells us that

$$
v_{o}=\frac{v_{s}}{\gamma(1-\beta \cos \theta)}
$$

where $\gamma=1 / \sqrt{ } 1-\beta^{2}$. Squaring both sides and rearranging the terms, we have

$$
(1-\beta \cos \theta)^{2}=\left(1-\beta^{2}\right) \frac{v_{s}^{2}}{v_{o}^{2}}=\left(1-\beta^{2} \sin ^{2} \theta-\beta^{2} \cos ^{2} \theta\right) \frac{v_{s}^{2}}{v_{o}^{2}}
$$

Eliminating $\beta \sin \theta$ by plugging Eq. (7) into Eq. (19), we obtain

$$
(1-\beta \cos \theta)^{2}=\left(1-\beta_{a p p, t}^{2}(1-\beta \cos \theta)^{2}-\beta^{2} \cos ^{2} \theta\right) \frac{v_{s}^{2}}{v_{o}^{2}}
$$


The rearrangement yields

$$
\left(1+\beta_{a p p t}^{2} \frac{v_{s}^{2}}{v_{o}^{2}}\right)(1-\beta \cos \theta)^{2}=\left(1-\beta^{2} \cos ^{2} \theta\right) \frac{v_{s}^{2}}{v_{o}^{2}}
$$

Cancellation by $1-\beta \cos \theta$ (not zero for non-zero masses) on both sides results in

$$
\left(1+\beta_{a p p, t}^{2} \frac{v_{s}^{2}}{v_{o}^{2}}\right)(1-\beta \cos \theta)=(1+\beta \cos \theta) \frac{v_{s}^{2}}{v_{o}^{2}}
$$

The rearrangement yields

$$
\beta_{r}=\beta \cos \theta=\frac{1+\left(\beta_{a p p, t}^{2}-1\right) \frac{v_{s}^{2}}{v_{o}^{2}}}{1+\left(\beta_{a p p, t}^{2}+1\right) \frac{v_{s}^{2}}{v_{o}^{2}}}
$$

in which all the factors on the RHS can be observationally obtained. Now, with br = b cosq, eliminating bapp;l by plugging Eq.(13) into Eq. (16) yields

$$
\begin{aligned}
\beta^{2} & =\left(1-\beta_{r}\right)^{2}\left[\beta_{a p p, t}^{2}+\frac{\beta_{r}^{2}}{\left(1-\beta_{r}\right)^{2}}\right] \\
& =\beta_{r}^{2}+\beta_{a p p, t}^{2}\left(1-\beta_{r}\right)^{2} \\
& =\frac{\left[1+\left(\beta_{a p p, t}^{2}-1\right) \frac{v_{s}^{2}}{v_{o}^{2}}\right]^{2}+4 \frac{v_{s}^{4}}{v_{o}^{4}} \beta_{a p p, t}^{2}}{\left[1+\left(\beta_{a p p, t}^{2}+1\right) \frac{v_{s}^{2}}{v_{o}^{2}}\right]^{2}}
\end{aligned}
$$

Hence the exact speed of the object is given by

$$
\beta=\frac{\sqrt{\left[1+\left(\beta_{a p p, t}^{2}-1\right) \frac{v_{s}^{2}}{v_{o}^{2}}\right]^{2}+4 \frac{v_{s}^{4}}{v_{o}^{4}} \beta_{a p p, t}^{2}}}{1+\left(\beta_{a p p, t}^{2}+1\right) \frac{v_{s}^{2}}{v_{o}^{2}}}
$$


with, if $\beta \neq 0$,

$$
\begin{aligned}
\theta & =\arccos \left(\frac{\beta_{r}}{\beta}\right) \\
& =\arccos \left(\frac{1+\left(\beta_{a p p, t}^{2}-1\right) \frac{v_{s}^{2}}{v_{o}^{2}}}{\sqrt{\left[1+\left(\beta_{a p p, t}^{2}-1\right) \frac{v_{s}^{2}}{v_{o}^{2}}\right]^{2}+4 \frac{v_{s}^{4}}{v_{o}^{4}} \beta_{a p p, t}^{2}}}\right)
\end{aligned}
$$

This is the result for relativistic Doppler effect.

\section{Conclusion}

With the concept of ALV $v_{a p p, l}$, we can determine the exact speed $v$ as well as the angle $q$ if both $\beta_{a p p, l}$ and $\beta_{a p p, t}$ are measured. However, all these results can only be guaranteed to hold for the radio sources which are at very low redshift $z \ll$ and stationary relative to the Earth's observer, and $\theta$ that can be generalized from $[0, \pi / 2]$ to $[0 / \pi]$. In order to generalize for all $z$ and $\beta \in$ $[0,1]$, we are suggested to follow Michal J.Chodorowski's treatment in his paper (Chodorowski 2005).

On the other hand, the principle of relativistic Doppler effect enables us to find the actual radial speed $v_{r}$, and thus determine $v$ and $\theta$, if the sources have the doppler-shifted effects. But the exact velocities of all jet sources might not be obtained via Doppler-shifted lines because the sources might not have such lines. Instead of considering just the Doppler effects or spectroscopic methods, we can consider further astrometric methods that might enable us to measure the radial velocity. The accuracies will increase with future space astrometry missions.

The relative change in the angular size of the jet might be confused with the evolutionary effects, so the changing angular extent is an infeasible method of measurement. An alternative measurement method, or a new astrometric method, is needed to obtain the exact velocity of the jet or the component. Yet it is important to note that if the jet is replaced by the stellar object which has the fixed size and is nearby, then the relative change in the angular size can ensure us to find the radial velocity of the object, as we can have the distance to the stellar object via parallaxes. So the astrometric radial velocity measurement is not confined to just jets, but extended to the stellar objects that are nearby. 


\section{Acknowledgements}

The topic presented here is based on ideas that were inspired by discussions in ASTR 4004/4005 Astrophysical Gas Dynamics class, which I took in 2010 as my third year research project. I feel tremendously indebted to Prof. Geoffrey Bicknell for his invaluable advice and patient supervision. Without his positive guidance and support I would not have understood as well the contemporary problem in Relativistic Jets and HzRG's.

I also thank PhD students and academics at the Research School of Astronomy \& Astrophysics who presented a lecture series to summer students in 2010. In particular, I appreciate the the opportunity to have been on the field trip to explore some of the premier radio and optical astronomy sites and telescopes in Australia. The trip was crucial to furthering my understanding of this research project. I also thank Graeme Blackman for kindly giving us a ride to Mount Stromlo every day.

Last but not least, I wish to thank Dr Paul Francis and the anonymous reviewer for their valuable comments and subsequent assistance in improving this manuscript.

\section{References}

Biretta, J. A., Sparks, W. B., Macchetto, F. 1999, ApJ, 520, 621

Chodorowski, M.J. 2005, AmJPh, 73, 639

Dravins D., Gullberg D., Lindegren L., Madsen S. 1999, ASPC, 185, 41

Madsen, S., Lindegren, L., Dravins, D. 1999, ASPC, 185, 77

Margon, B. 1984, ARAA, 22, 507

Mirabel, I.F., Rodriguez, L.F. 1998, Nature, 392, 673

Shu, F.H. 1982, The Physical Universe: An Introduction to Astronomy, Univ. Science Books, Mill Valley, CA, p.313 